\title{
Phosphorus-Free Membrane Lipids of Sinorhizobium meliloti Are Not Required for the Symbiosis with Alfalfa but Contribute to Increased Cell Yields Under Phosphorus-Limiting Conditions of Growth
}

\author{
Isabel M. López-Lara, ${ }^{1}$ Jun-Lian Gao, ${ }^{1}$ María José Soto, ${ }^{2}$ Alhondra Solares-Pérez, ${ }^{1}$ Barbara \\ Weissenmayer, ${ }^{1}$ Christian Sohlenkamp, ${ }^{1}$ George P. Verroios, ${ }^{3}$ Jane Thomas-Oates, ${ }^{3}$ and Otto Geiger ${ }^{1}$ \\ ${ }^{1}$ Centro de Ciencias Genómicas, Universidad Nacional Autónoma de México, Apdo. Postal 565-A, Cuernavaca, Morelos, \\ CP62210, Mexico; '²Departamento de Microbiología del Suelo y Sistemas Simbióticos. Estación Experimental del Zaidín. \\ CSIC. Profesor Albareda, 1, 18008 Granada, Spain; ${ }^{3}$ Department of Chemistry, University of York, Heslington, York, YO10 \\ 5DD, U.K., and formerly Michael Barber Centre for Mass Spectrometry, Department of Chemistry, UMIST, PO Box 88, \\ Manchester M60 1QD, U.K.
}

Submitted 21 February 2005. Accepted 2 May 2005.

\begin{abstract}
The microsymbiont of alfalfa, Sinorhizobium meliloti, possesses phosphatidylglycerol, cardiolipin, phosphatidylethanolamine, and phosphatidylcholine as major membrane phospholipids, when grown in the presence of sufficient accessible phosphorus sources. Under phosphate-limiting conditions of growth, $S$. meliloti replaces its phospholipids by membrane lipids that do not contain any phosphorus in their molecular structure and, in S. meliloti, these phosphorus-free membrane lipids are sulphoquinovosyl diacylglycerols (SL), ornithine-containing lipids (OL), and diacylglyceryl- $N, N, N$-trimethylhomoserines (DGTS). In earlier work, we demonstrated that neither SL nor OL are required for establishing a nitrogen-fixing root nodule symbiosis with alfalfa. We now report the identification of the two structural genes $b t a A$ and $b t a B$ from $S$. meliloti required for DGTS biosynthesis. When the sinorhizobial btaA and btaB genes are expressed in Escherichia coli, they cause the formation of DGTS in this latter organism. A btaA-deficient mutant of $S$. meliloti is unable to form DGTS but can form nitrogen-fixing root nodules on alfalfa, demonstrating that sinorhizobial DGTS is not required for establishing a successful symbiosis with the host plant. Even a triple mutant of $S$. meliloti, unable to form any of the phosphorusfree membrane lipids SL, OL, or DGTS is equally competitive for nodule occupancy as the wild type. Only under growth-limiting concentrations of phosphate in culture media did mutants that could form neither OL nor DGTS grow to lesser cell densities.
\end{abstract}

Rhizobial bacteria are able to interact with leguminous plants leading to the formation of a nitrogen-fixing root nodule symbiosis. During the onset of the symbiosis, a complex exchange of signals occurs between the bacteria and the legume;

Corresponding author: O. Geiger; E-mail: otto@ccg.unam.mx; Telephone: +52-7773-290815; Fax: +52-7773-175581.

Current address for B. Weissenmayer: Institute of Biology-Microbiology, Free University Berlin, Königin-Luise-Strasse 12-16, D-14195 Berlin, Germany. rhizobia enter root hairs and underlying cells via infection threads from which they are eventually released and are able to enter cortical cells via endocytosis. This series of events results in a unique cytoplasmic organelle called the symbiosome, which consists of modified bacteria (bacteroids) surrounded by a membrane of plant origin, called the peribacteroid membrane. In the established symbiosis, the bacterial microsymbiont provides reduced nitrogen sources to the plant and, in return, the plant macrosymbiont supplies organic carbon sources to the bacteria. Besides nitrogen, phosphorus is often one of the limiting elements for efficient plant growth in soil, and many plants form an additional symbiosis with mycorrhizal fungi in order to guarantee them an adequate supply of phosphorus. When plants are grown in the presence of sufficient phosphate, phosphorus-free galactolipids, such as monogalactosyldiacylglycerol and digalactosyldiacylglycerol (DGDG), are restricted to the chloroplast membranes. In contrast, under conditions of phosphate deprivation, the amount of DGDG increases strongly, and it occurs in extraplastidic membranes such as the plasma membrane as well (Dörmann and Benning 2002). Recent evidence suggests that peribacteroid membranes of soybean and Lotus contain DGDG as a major membrane lipid independently of whether plants were grown under phosphatesufficient or -limiting conditions (Gaude et al. 2004). In some bacteria under phosphorus-limiting conditions, membrane phospholipids are also at least partially replaced by lipids containing no phosphorus, as demonstrated in Bacillus subtilis (Minnikin et al. 1972), Pseudomonas diminuta (Minnikin et al. 1974), Pseudomonas fluorescens (Minnikin and Abdolrahimzadeh 1974), and Rhodobacter sphaeroides (Benning et al. 1995). In Sinorhizobium (Rhizobium) meliloti, these phosphorus-free lipids are sulfoquinovosyl diacylglycerol (SL), ornithine-containing lipids (OL), and diacylglyceryl- $N, N, N$-trimethylhomoserine (DGTS) (Geiger et al. 1999). Therefore, plants as well as some bacteria can replace their phospholipids with membrane lipids that do not contain any phosphorus, and this replacement is interpreted as a response to phosphorus-limiting stress conditions in which less phosphorus is consumed for the formation of membranes.

The status of available phosphorus within the nodule and specifically for bacteroids has been unclear. Alkaline phospha- 
tase is present at high levels in Rhizobium tropici bacteroids in phosphorus-sufficient plants leading to the suggestion that inorganic phosphorus levels must be low enough to trigger a phosphate stress response (Al-Niemi et al. 1997a). In addition, mutants of $S$. meliloti deficient in the high-affinity phosphate transporter PhoCDET form nodules that contain few bacteria and are unable to fix nitrogen, probably due to their failure to obtain sufficient phosphorus during the infection process (Bardin et al. 1996), which seemed to support further the idea that rhizobial bacteroids and rhizobia during the infection process might be limited for phosphorus.

The glycerophospholipids phosphatidylglycerol, cardiolipin, phosphatidylethanolamine, and phosphatidylcholine are the
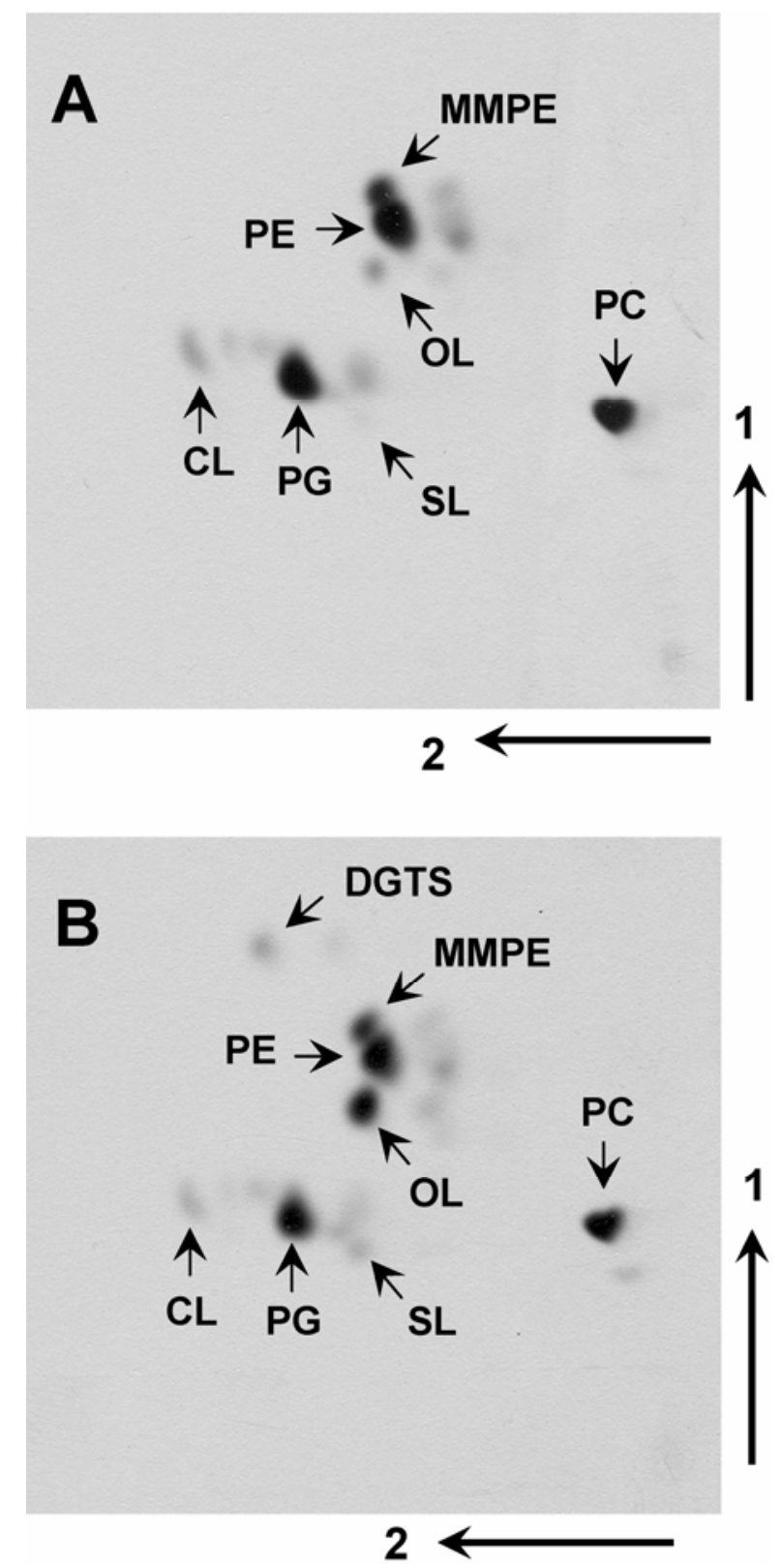

Fig. 1. Separation of $\left[{ }^{14} \mathrm{C}\right]$ acetate-labeled lipids from Sinorhizobium meliloti 1021. Lipid extracts from $\mathbf{A}$, the wild type used in our laboratory, Sm1021our and B, strain Sm1021sequenced, the genome of which had been sequenced, were separated by two-dimensional thin-layer chromatography after growth on complex tryptone yeast extract medium. The lipids phosphatidylcholine (PC), phosphatidylethanolamine (PE), monomethylphosphatidylethanolamine (MMPE), phosphatidylglycerol (PG), cardiolipin (CL), sulfolipid (SL), ornithine-containing lipid (OL), and diacylglyceryl$\mathrm{N}, \mathrm{N}, \mathrm{N}$-trimethylhomoserine (DGTS) are indicated. major components of the $S$. meliloti membrane if the organism has been cultured in the presence of sufficient phosphate. However, when phosphorus supplies become growth-limiting, $S$. meliloti replaces its phospholipids with membrane-forming lipids that do not contain phosphorus, SL, OL, and DGTS (Geiger et al. 1999). If bacteroids suffered from phosphorus limitation, one might have expected that the sinorhizobial phosphorus-free lipids SL, OL, and DGTS would be formed within bacteroids and that the inability to form one or several of these lipids might impair the symbiotic performance of such mutants.

Whereas, in our earlier work, we characterized mutants of $S$. meliloti deficient in SL (Weissenmayer et al. 2000) or OL (Gao et al. 2004; Weissenmayer et al. 2002), we have now investigated mutants of $S$. meliloti unable to form DGTS as well as mutants lacking two or all three of these lipids with regard to their symbiotic behavior or growth under free-living conditions. Here, we show that none of these phosphorus-free membrane lipids is required for a successful and efficient symbiosis of $S$. meliloti with its host plant alfalfa. However, with mutants of $S$. meliloti unable to form OL and DGTS, a lower growth yield is obtained under phosphate-limiting conditions than with wild type.

\section{RESULTS}

\section{S. meliloti 1021 strain, the genome} of which has been sequenced, displays

the membrane lipid profile of a phosphorus-limited strain.

During a recent transcriptional analysis of S. meliloti 1021, of which the genome has been sequenced (Sm1021sequenced), it was observed that this strain suffers from phosphate starvation even in the presence of sufficient available phosphate in the growth medium, due to a mutation in the pstC gene (Krol and Becker 2004). We reported earlier that S. meliloti 1021 commonly used in our laboratory (Sm1021our) presents different lipid profiles when grown on minimal media with relatively high $(1.3 \mathrm{mM})$ concentrations of inorganic phosphate or with growth-limiting $(0.02 \mathrm{mM})$ concentrations of phosphate, and there was no indication that Sm1021our suffered from phosphate starvation when grown in the presence of $1.3 \mathrm{mM}$ phosphate. Both strains, Sm1021our and Sm1021sequenced, were confirmed to be sensitive to rifampicin and resistant to streptomycin (data not shown), as expected for S. meliloti 1021, and therefore, we reinvestigated the lipid profiles formed by Sm1021our (Fig. 1A) and by Sm1021sequenced (Fig. 1B) after growth on medium in the presence of high phosphate. In Sm1021our, mainly the phospholipids cardiolipin (CL), phosphatidylglycerol (PG), phosphatidylethanolamine (PE), monomethylphosphatidylethanolamine (MMPE), and phosphatidylcholine (PC) were formed and only minor amounts of the phosphorus-free membrane lipids SL and OL (1.2 and $1.4 \%$ of total lipids, respectively) (Fig. 1A), confirming the results reported earlier (Geiger et al. 1999). In contrast, in Sm1021sequenced, the relative amounts of phospholipids were reduced, whereas SL and OL (2.1 and $17.3 \%$ of total lipids, respectively) were increased (Fig. 1B). Most notably, Sm1021sequenced forms DGTS (2.4\% of total lipids) in media with high concentrations of phosphate, a lipid that is formed by Sm1021our only when phosphate concentrations become growth-limiting. Analysis of protein expression profiles (Djordjevic et al. 2003) as well as of transcripts (Krol and Becker 2004) had already suggested that Sm1021sequenced is phosphate-stressed, even when grown in the presence of sufficient available phosphate, a fact that is reflected in its lipid profile as well. Therefore, strain Sm1021sequenced is not a good choice for studying phosphate limitation. The $S$. meliloti 1021 strain used in our 
studies (Sm1021our) is not stressed for phosphorus when grown in the presence of $1.3 \mathrm{mM}$ phosphate, and only Sm1021our, which is subsequently termed Sm1021, or strains derived from it were used in the remaining studies.

\section{SMc01848 is required for DGTS formation in $\mathrm{S}$. meliloti.}

In $S$. meliloti, when phosphate is growth-limiting, a de novo biosynthesis of DGTS lipids is observed (Geiger et al. 1999). The structural genes responsible for DGTS biosynthesis were identified originally in Rhodobacter sphaeroides and were named btaA and btaB (Klug and Benning 2001). Searching with the rhodobacterial BtaA protein sequence (416 amino acids) in the $S$. meliloti 1021 genome, we found that SMc01848 (416 amino acids) shows 50\% identity and 63\% similarity to $R$. sphaeroides BtaA. Immediately downstream of gene smc01848, and forming an operon with it, is smc01847 (with its start codon preceding and overlapping the stop codon of smc01848), which encodes a protein of 221 amino acids showing $52 \%$ identity and $66 \%$ similarity to BtaB from $R$. sphaeroides (210 amino acids). In order to find out if SMc01848 is required for DGTS biosynthesis, a mutant that lacks an internal fragment of the smc01848 gene was created in S. meliloti 1021 (Fig. 2; discussed below) and was designated DGTS1. When S. meliloti 1021 wild type (Fig. 3, lane 1) or mutant DGTS1 (Fig. 3, lane 2) were grown on high (1.3 $\mathrm{mM}$ ) phosphate-containing minimal medium, no difference in lipid patterns is observed and, under such conditions, mainly phospholipids and only minor amounts of OL or SL are formed (Geiger et al. 1999). After growth under phosphatelimiting conditions, the wild type displays a rather different lipid pattern, with DGTS as the major membrane lipid (Geiger et al. 1999; Fig. 3, lane 3). However, when mutant DGTS1 is grown under phosphate limitation no DGTS is formed (Fig. 3, lane 4). Introduction of plasmid pJG03, which carries smc01848 and smc01847, restores DGTS formation in mutant DGTS1 under phosphate-limiting conditions of growth (Fig. 3, lane 6), which is not the case when DGTS1 harbors the empty vector pRK404 (Fig. 3, lane 5). We therefore conclude that smc01848 and smc01847 are the btaA and btaB genes of $S$. meliloti.

As the lack of DGTS seemed to be compensated for by the specific increase in some other lipids (Fig. 3), the lipid patterns of phosphate-limited S. meliloti wild type, DGTS-deficient null mutant DGTS1, and the btaA- and btaB-complemented mutant DGTS $1 \times$ pJG03 were also compared after two-dimensional thin-layer chromatography (TLC) separation and were quantified (Table 1 ). The $S$. meliloti 1021 wild type shows essentially the lipid composition reported earlier (Geiger et al. 1999). However, in the DGTS1 mutant, the lack of DGTS seems to be compensated for by a relative increase in some other lipids (PC, SL, and OL) (Table 1). Notably, in a DGTSdeficient $S$. meliloti strain, OL comprises around half of all membrane lipids under conditions of phosphorus limitation. Expression of $b t a A$ and $b t a B$ (from pJG03) in the DGTS1 background essentially restores the lipid composition observed in the wild type (Table 1), whereas this is not the case if the DGTS1 mutant harbors the empty vector (pRK404).

\section{Expression of the sinorhizobial $b t a A$ and $b t a B$ genes in Escherichia coli leads to DGTS formation.}

Strains of E. coli do not possess genes homologous to btaA and $b t a B$ (López-Lara et al. 2003) and therefore can be used as hosts for studying the functionality of btaA and $b t a B$ gene products. The coding regions of the sinorhizobial btaA and $b t a B$ genes were cloned in the $E$. coli expression vector pET9a to generate plasmid pIML05, in order to investigate whether they were sufficient to direct DGTS biosynthesis when heterologously expressed in E. coli. Overexpression of btaA and btaB in E. coli BL21(DE3) pLysS pIML05 (Fig. 4A, lanes 3 and 5) caused the formation of a compound that migrated with the same relative mobility as DGTS produced in S. meliloti 1021 under phosphate-limiting conditions (Fig. 4A, lane 1). While the DGTS-like compound is formed in cells grown at 30 or at $37^{\circ} \mathrm{C}$ (Fig. 4A, lanes 3 or 5), lipid quantification showed that, in cells grown at $30^{\circ} \mathrm{C}$, DGTS-like compounds account for $3.5 \%$ of total lipids whereas, in cells grown at $37^{\circ} \mathrm{C}$, they account for $1.5 \%$, indicating better functioning of the BtaA- and BtaB-catalyzed reactions at $30^{\circ} \mathrm{C}$.

The structures of the lipids produced by E. coli BL21(DE3) pLysS carrying either pET9a or pIML05 were studied, using electrospray mass spectrometry (ES-MS) in the positive ion mode. Information on the molecular masses of the species present was obtained by the use of ES-MS and the structures were determined using ES collision-induced dissociation (CID) MS/MS. In the spectrum obtained from the lipid extract of the strain that harbors plasmid pIML05 (Fig. 4B), intense, singly charged ions were observed at $\mathrm{m} / \mathrm{z} 738$ and 764, together with less intense ions at $\mathrm{m} / \mathrm{z} 690,704,710$, and 718. These ions were selected for collisional activation and MS/MS analysis. Only the two intense ions $(\mathrm{m} / \mathrm{z}, 738$ and 764) gave fragment ions diagnostic of DGTS. The ion at $\mathrm{m} / z 764$ was induced to dissociate into two major ions with $\mathrm{m} / \mathrm{z}, 500$ and 236. These

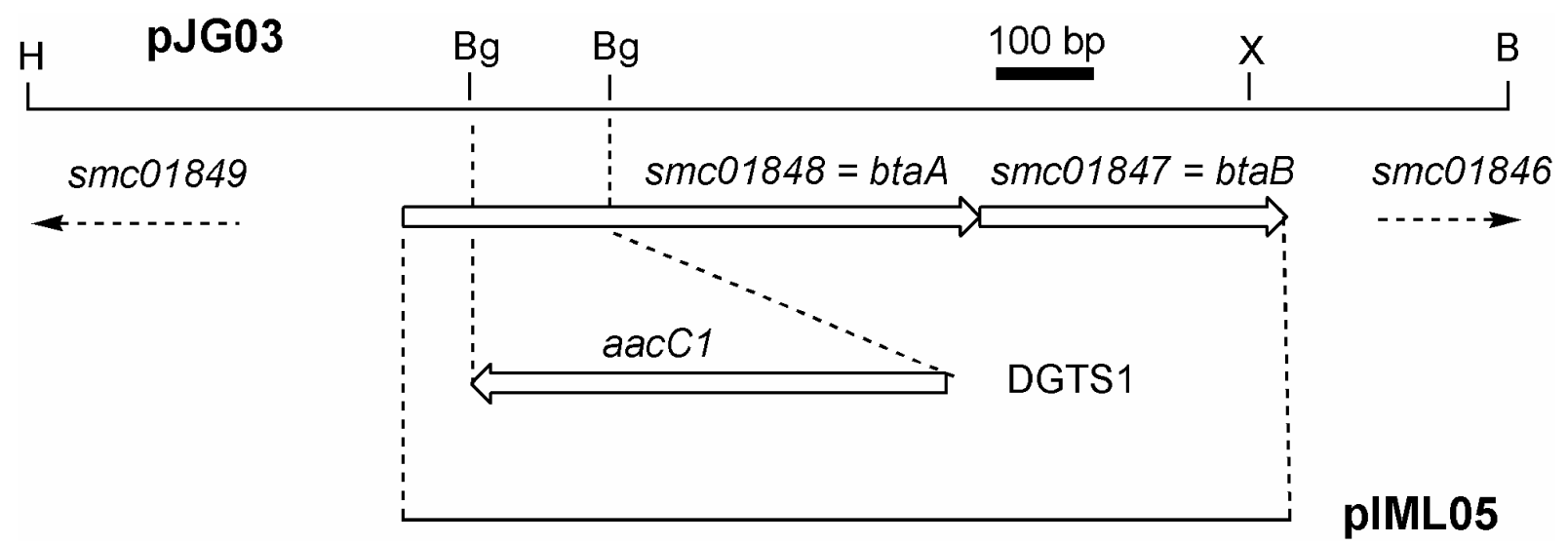

Fig. 2. Physical and restriction map of the genomic sinorhizobial DNA region containing $s m c 01848$ (btaA) and $s m c 01847$ (btaB), the structural genes for biosynthesis of diacylglyceryl- $N, N, N$-trimethylhomoserines (DGTS). The location of the amplified fragments cloned in pJG03 or in pIML05 is indicated. The replacement of the internal 316-bp BglII fragment of smc01848 by a gentamicin resistance cassette (aacC1, gene for gentamicin acetyl transferase) in mutant DGTS1 is indicated. Restriction sites: $\mathrm{B}=$ Bam HI, $\mathrm{Bg}=B g l \mathrm{II}, \mathrm{H}=$ HindIII, and X $=$ XhoI. 
two sequential losses of $264 \mathrm{Th}$ can be explained because this DGTS has two C18:1 fatty acyl chains. The fragment ion observed with $m / z, 482$ corresponds to the loss of water from the ion with $\mathrm{m} / z, 500$. Finally, the ion observed at $\mathrm{m} / \mathrm{z}, 144$ corresponds to the head group of the DGTS.

CID of the ion with $\mathrm{m} / \mathrm{z} 738$ generated four major fragment ions with $\mathrm{m} / \mathrm{z}$ values of $500,474,236$, and 456 . The loss of 238 and 264 Th from the precursor ion to yield the product ions at $\mathrm{m} / \mathrm{z}, 500$ and 474 can be attributed to the sequential loss of the two fatty acids. The loss of $238 \mathrm{Th}$ corresponds to the loss of a C16:0 fatty acyl chain, while the loss of 264 Th corresponds again to the loss of a C18:1 fatty acyl chain. The ion with $\mathrm{m} / \mathrm{z}, 236$ is the product of cleavage of both fatty acids. Finally, the $m / z 456$ ion is the result of the elimination of a molecule of water (18 Th) from the $m / z 474$ ion. The ion with $m / z$ 144 is observed again, confirming that the ion at $m / z, 738$ does indeed arise from DGTS.

In comparison, the major ion in the spectrum of the strain harboring just the vector plasmid (pET9a) was observed at $m / z$ 188. There is no ion with $\mathrm{m} / \mathrm{z}, 738$, but there is an ion at $\mathrm{m} / \mathrm{z}$ 765, just 1 Th higher than the DGTS ion described above. This

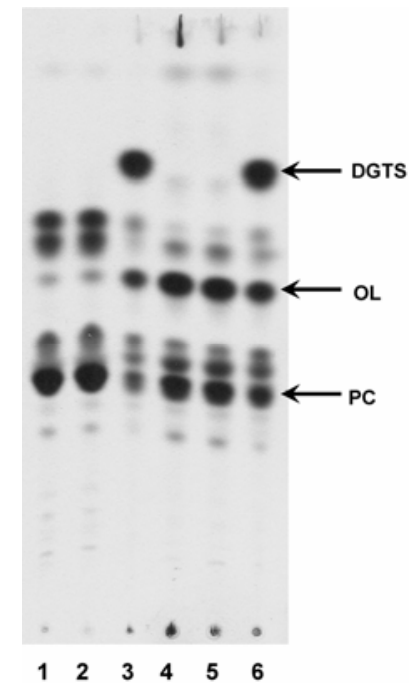

Fig. 3. The sinorhizobial mutant DGTS1 is defective in diacylglyceryl$N, N, N$-trimethylhomoserines (DGTS) formation. Sinorhizobium meliloti strains were radiolabeled during growth on Sherwood minimal medium with high $(1.3 \mathrm{mM})$ concentrations of phosphate (lanes 1 and 2) or with growth-limiting ( $0.02 \mathrm{mM})$ concentrations of phosphate (lanes 3 to 6 ), and lipids were separated by one-dimensional thin-layer chromatography. The strains analyzed were S. meliloti wild-type 1021 (lanes 1 and 3), S. meliloti DGTS1 (lanes 2 and 4), and S. meliloti DGTS1 containing either pRK404 (lane 5) or pJG03 (lane 6). ion was chosen for fragmentation along with the most abundant ions in the spectrum. However, no diagnostic fragment ions for DGTS were observed in the tandem mass spectra obtained from any of the species produced by this strain (data not shown).

\section{Phosphorus-free membrane lipids \\ of $S$. meliloti are required neither for symbiosis with alfalfa nor for competitiveness.}

In addition to SL-deficient SLD12 and OL-deficient ORLD8, a mutant unable to synthesize DGTS (DGTS1) as well as the three possible double mutants (SL- and DGTS-deficient SD1, OL- and SL-deficient OS1, OL- and DGTS-deficient OD1), and the triple mutant SOD1, unable to synthesize OL, SL, and DGTS, were constructed (Table 2). In order to study if phosphorus-free membrane lipids may play a role in the symbiotic association of $S$. meliloti and its host alfalfa, aseptically grown alfalfa seedlings were inoculated with the wild type, the different mutants deficient in phosphorus-free membrane lipids (SLD12, ORLD8, DGTS1, SD1, OS1, OD1, or SOD1), or treated with water as a control. The plant mineral solution used in the experiments contained $0.7 \mathrm{mM}$ phosphate, a relatively high concentration, which was chosen to prevent adverse effects on sinorhizobial mutant strains as long as they were outside the host plant. Plants inoculated with any of the eight $S$. meliloti strains formed nodules, while no nodules were formed on water-treated plants. Furthermore, there was no significant difference in the number of nodules developing over time on the roots of plants treated with wild type or mutant bacteria (data not shown). Lipid analysis of bacteria reisolated from the nodules to confirm the bacterial phenotypes showed the same membrane lipid composition as found in cells used for inoculation. These observations suggest that there are no adverse effects on the development and function of nodules derived from $S$. meliloti strains lacking any of the phosphorusfree membrane lipids.

Occasionally, a slight increase in nodule formation efficiency has been observed for the SOD1 mutant when compared with the wild-type strain. However, in general no big differences in nodulation kinetics by the two strains were observed under our experimental conditions (Fig. 5A). Likewise, results obtained in competition assays indicate that the SOD1 mutant has a similar competitive ability as the wild type (Fig. 5B). Therefore, the lack of any or of all of the phosphorus-free membrane lipids in $S$. meliloti does not alter the competitive ability of this bacterium.

DGTS or OL are required for a wild-typelike growth yield in phosphate-limiting media.

We have reported previously that mutants of $S$. meliloti 1021 deficient in SL (Weissenmayer et al. 2000) or mutants deficient

Table 1. Membrane lipid composition ${ }^{\mathrm{a}}$

\begin{tabular}{|c|c|c|c|c|c|}
\hline \multirow[b]{2}{*}{ Lipid $^{b}$} & \multicolumn{5}{|c|}{ Composition ( $\%$ of total ${ }^{14} \mathrm{C}$ ) } \\
\hline & Wild type & DGTS1 & DGTS1 $\times$ pJG03 & DGTS1 × pRK404 & SOD1 \\
\hline $\mathrm{PG}+\mathrm{CL}$ & $5.9 \pm 1.0$ & $7.4 \pm 1.0$ & $10.2 \pm 1.9$ & $10.2 \pm 3.4$ & $31.0 \pm 1.7$ \\
\hline $\mathrm{PE}+\mathrm{MMPE}$ & $2.6 \pm 1.2$ & $2.5 \pm 0.5$ & $3.5 \pm 0.9$ & $3.8 \pm 1.9$ & $25.6 \pm 6.3$ \\
\hline $\mathrm{PC}$ & $1.3 \pm 0.1$ & $17.0 \pm 3.7$ & $8.4 \pm 1.6$ & $20.1 \pm 7.2$ & $43.4 \pm 4.8$ \\
\hline SL & $8.9 \pm 0.6$ & $19.0 \pm 3.1$ & $12.4 \pm 2.8$ & $19.3 \pm 1.6$ & n.d. \\
\hline $\mathrm{OL}$ & $14.0 \pm 1.7$ & $54.1 \pm 2.5$ & $17.2 \pm 3.6$ & $46.5 \pm 12.3$ & n.d. \\
\hline DGTS & $67.4 \pm 1.3$ & n.d. ${ }^{\mathrm{c}}$ & $48.3 \pm 5.6$ & n.d. & n.d. \\
\hline
\end{tabular}

a Composition of Sinorhizobium meliloti 1021 wild type, diacylglyceryl trimethylhomoserine (DGTS)-deficient btaA mutant (DGTS1), btaA mutant (DGTS1) complemented with the $b t a A$ and $b t a B$ genes (DGTS1 1 pJG03) or containing an empty broad-host-range vector (DGTS1 $\times$ pRK404) as well as triple mutant ( $s q d B-$, olsA-, btaA-deficient) SOD1 after growth on low $(0.02 \mathrm{mM})$ phosphate-containing minimal medium. The values shown are mean values \pm standard deviation derived from three independent experiments.

${ }^{\mathrm{b}} \mathrm{PG}=$ phosphatidylglycerol, $\mathrm{CL}=$ cardiolipin, $\mathrm{PE}=$ phosphatidylethanolamine, $\mathrm{MMPE}=$ monomethylphosphatidylethanolamine, $\mathrm{PC}=$ phosphatidylcholine, $\mathrm{SL}$ $=$ sulfolipid, and $\mathrm{OL}=$ ornithine-containing lipid.

${ }^{\mathrm{c}}$ n.d. $=$ not detected. 
in OL (Gao et al. 2004; Weissenmayer et al. 2002) show no significant growth phenotype in comparison to the wild-type strain when grown in phosphate-limiting media. Furthermore, under those conditions, mutants reached the same final optical and, therefore, cell density as the wild type. We have now followed the growth of wild type, of each of the three single mutants (SLD12, ORLD8, or DGTS1), of the three double mutants
(SD1, OS1, or OD1), and of the triple mutant SOD1 in minimal medium containing sufficient or growth-limiting amounts of phosphate. In medium with sufficient inorganic phosphate $(1.3 \mathrm{mM} \mathrm{Pi})$, there are no significant differences in growth between the wild type and any one of the mutants (data not shown). When the eight different strains were grown under phosphate-limiting conditions, no significant differences were

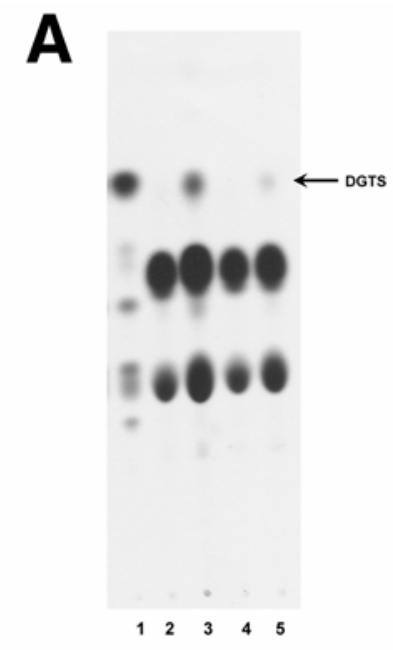

B

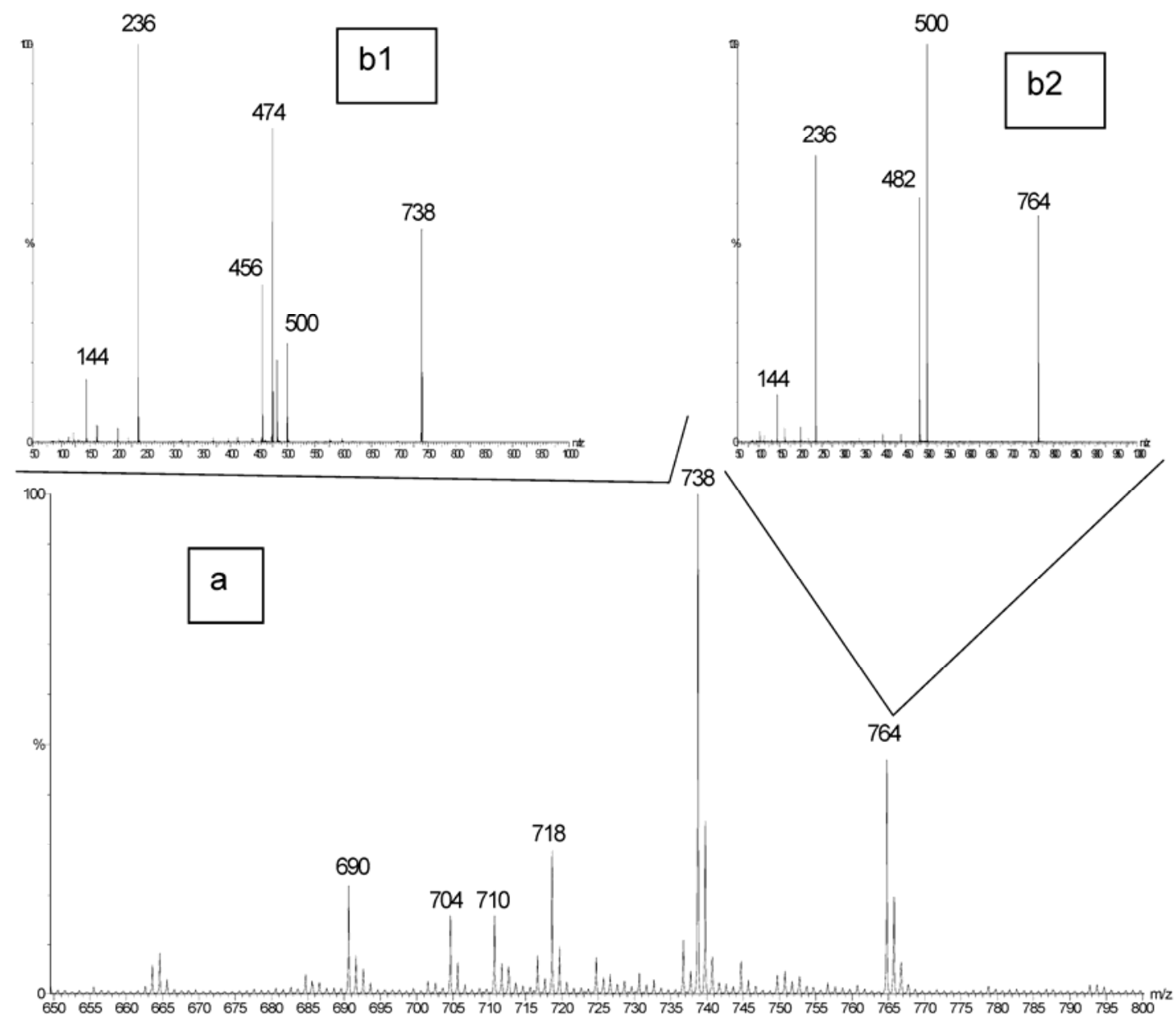

Fig. 4. Diacylglyceryl- $N, N, N$-trimethylhomoserines formation in Escherichia coli. A, Lipids from E. coli BL21(DE3) pLysS containing either pET9a (lanes 2 and 4) or pIML05 (lanes 3 and 5) were radiolabeled during growth on Luria-Bertani medium at 30 (lanes 2 and 3) or 37 $7^{\circ} \mathrm{C}$ (lanes 4 and 5) and were separated by one-dimensional thin-layer chromatography. As reference, radiolabeled lipids obtained from Sinorhizobium meliloti 1021 grown under phosphorus-limiting conditions were separated (lane 1). B, Electrospray (a) and tandem mass spectra (b1 and b2) of a lipid extract from E. coli BL21(DE3) harboring plasmid pIML05. 
observed during the exponential phase of growth (Fig. 6 and data not shown). However, mutants OD1 and SOD1 reached lower final optical densities than the wild type or the other five mutant strains (Fig. 6). While the wild type reached a final optical density close to 0.6 under phosphate-limiting conditions, mutants OD1 and OSD1 reached final optical densities of only around 0.45 . When comparing the means of the final cell densities of the wild type with those of mutant OD1 or of mutant SOD1, they are significantly different, at a confidence level of
$>99 \%$, using Student's $t$ test. Therefore, the ability to form DGTS or OL ensures a higher growth yield under phosphatelimiting growth conditions. The lipid composition of the SOD1 mutant was analyzed after growth under phosphate-limiting conditions and was found to be significantly different from the lipid composition of the wild-type 1021 grown under the same conditions (Table 1). Under phosphate-limiting growth conditions, about 80 to $90 \%$ of the membrane lipids of wild-type 1021 are phosphorus-free membrane lipids (Table 1) (Geiger

Table 2. Bacterial strains and plasmids used in this study

\begin{tabular}{|c|c|c|}
\hline Strain or plasmid & Relevant characteristics $^{\mathbf{a}}$ & Reference or source \\
\hline Sinorhizobium meliloti 1021 & SU47 str-21 & Meade et al. 1982 \\
\hline S. meliloti 1021 sequenced & $\begin{array}{l}\text { "Wild type" strain from which the genome has been sequenced, pstC- } \\
\text { deficient }\end{array}$ & $\begin{array}{l}\text { Galibert et al. 2001; Krol and Becker 2004; } \\
\text { The EEZ (Granada) strain collection }\end{array}$ \\
\hline $\begin{array}{l}\text { S. meliloti } 1021 \text { our } \\
\text { Sm } 1021 \text { derivatives }\end{array}$ & Wild type used throughout this study & The Leiden University strain collection \\
\hline SLD11 & $s q d B:: k a n$ & Weissenmayer et al. 2000 \\
\hline SLD12 & sqdB::kan & This study \\
\hline ORLD8 & olsA::spec & Weissenmayer et al. 2002 and this work \\
\hline DGTS1 & btaA::gm & This study \\
\hline SD1 & Deficient in SL and DGTS, sqdB::kan; btaA::gm & This study \\
\hline OS1 & Deficient in OL and SL, olsA::spec; sqdB::kan & This study \\
\hline OD1 & Deficient in OL and DGTS, olsA::spec; btaA::gm & This study \\
\hline SOD1 & $\begin{array}{l}\text { Triple mutant deficient in SL, OL and DGTS, sqdB::kan; olsA::spec; } \\
\quad \text { btaA::gm }\end{array}$ & This study \\
\hline \multicolumn{3}{|c|}{ 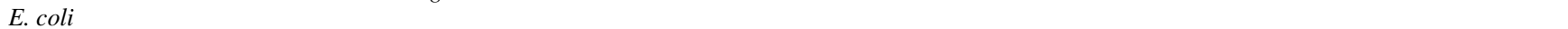 } \\
\hline DH5 $\alpha$ & recA1, Ф80 lacZ $\Delta M 15$ & Hanahan et al. 1983 \\
\hline BL21(DE3).pLysS & Expression strain & Studier et al. 1990 \\
\hline \multicolumn{3}{|l|}{ Plasmids } \\
\hline pHY109 & $\begin{array}{l}\text { Spectinomycin resistance-conferring } \Omega \text { interposon cloned in the EcoRI } \\
\text { restriction sites of tetracycline-resistant IncP plasmid pCHO341 }\end{array}$ & Østerås et al. 1998 \\
\hline pRK2013 & Helper plasmid, kanamycin resistant & Figurski and Helinski, 1979 \\
\hline pRK404 & Broad host range vector, tetracycline resistant & Ditta et al. 1985 \\
\hline pMP3510 & Broad-host-range vector, tetracycline resistant & Spaink et al. 1995 \\
\hline pBBR1MCS & Broad-host-range vector, chloramphenicol resistant & Kovach et al. 1994 \\
\hline $\mathrm{pAC} \Omega-\mathrm{Gm}$ & Broad-host-range vector containing gentamicin resistance cassette & Schweizer 1993 \\
\hline pET9a & Expression vector, kanamycin resistant & Studier et al. 1990 \\
\hline pK18mobsacB & Suicide vector, kanamycin resistant & Schäfer et al. 1994 \\
\hline pUC18/pUC19 & Cloning vectors, carbenicillin resistant & Yanisch-Perron et al. 1985 \\
\hline pGUS3 & pnfeD-gusA translational fusion in pBI101 (Clontech), kanamycin resistant & García-Rodríguez and Toro 2000 \\
\hline pBW63 & olsA-containing DNA, $P f u$-amplified $3.2-\mathrm{kb} X b a \mathrm{I}-K p n \mathrm{I}$ insert in pUC19 & Weissenmayer et al. 2002 \\
\hline pBW71 & 5.2-kb XbaI-KpnI olsA::spec-containing insert in pUC19 & This study \\
\hline pBW76 & 5.2-kb XbaI-KpnI olsA::spec-containing insert in pMP3510 & This study \\
\hline pBW89 & HindIII and Bam HI fragment containing btaA and btaB in pUC19 & This study \\
\hline pJG03 & HindIII and Bam HI fragment containing btaA and btaB in pRK404 & This study \\
\hline pJG26 & HindIII and Bam HI fragment containing btaA and btaB in pBBR1MCS & This study \\
\hline pJG28 & pJG26 with a gentamicin cassette in btaA & This study \\
\hline pJG30 & $\begin{array}{l}\text { XhoI fragment from pJG28, containing the gentamicin-inactivated btaA, in } \\
\text { pK18mobsacB }\end{array}$ & This study \\
\hline pIML05 & pET9a carrying btaA and btaB (smc01848 and smc01847) & This study \\
\hline
\end{tabular}

${ }^{\text {a }} \mathrm{SL}=$ sulfolipid, DGTS = diacylglyceryl trimethylhomoserine, and $\mathrm{OL}=$ ornithine-containing lipid.
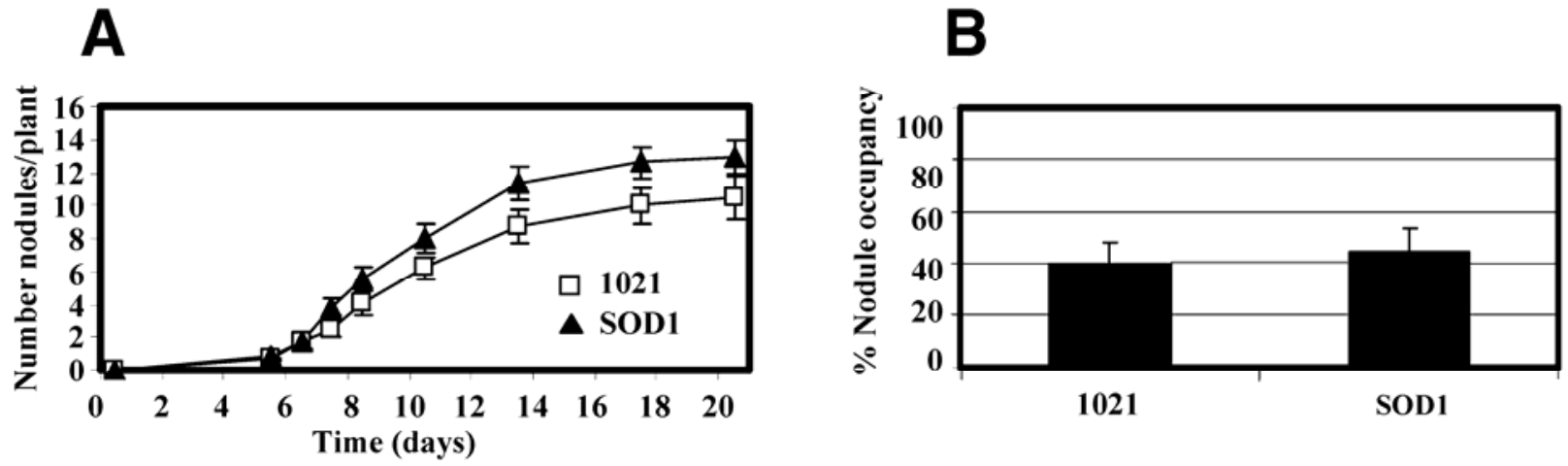

Fig. 5. Symbiotic phenotype of Sinorhizobium meliloti mutant SOD1. A, Nodulation kinetics of S. meliloti 1021 and SOD1 expressed as mean number of nodules formed per plant. Error bars represent standard errors at $95 \%$ confidence interval. B, Competition assay. Data represent the percentage of white nodules occupied by strains 1021 and SOD1 after inoculation with 1:1 mixtures of $1021 \times 1021$ (pGUS3) and SOD1 $\times 1021$ (pGUS3). Error bars are indicated at a confidence level of $95 \%$. 
et al. 1999), while all the membrane lipids detected in the triple mutant SOD1 are the well-known phospholipids PC, PE, $\mathrm{PG}$, and CL.

\section{DISCUSSION}

Two structural genes (btaA and $b t a B$ ) required for DGTS biosynthesis had originally been identified in Rhodobacter sphaeroides (Klug and Benning 2001). S. meliloti 1021 wild type produces large amounts of DGTS when grown under phosphorus-limiting conditions (Geiger et al. 1999). In contrast, a knock-out mutant of $S$. meliloti in smc01848 (DGTS1) is unable to form DGTS under phosphorus limitation. If the mutant DGTS1 is complemented with a plasmid that carries smc01848 and smc01847, DGTS biosynthesis is restored in $S$. meliloti. Expression of smc01848 and smc01847 in E. coli causes the formation of DGTS in this latter organism, demonstrating that, besides smc01848 and smc01847, no other genes not occurring in E. coli are required for DGTS biosynthesis. Taken together, these results show that smc01848 and $s m c 01847$ are the sinorhizobial structural genes $b t a A$ and $b t a B$, which are required for DGTS biosynthesis.

Based on earlier data (Al-Niemi et al. 1997a; Bardin et al. 1996), it was suggested that rhizobia in their developed bacteroidal form might be limited for phosphate. In free-living conditions under phosphorus limitation, more than $80 \%$ of all membrane lipids in $S$. meliloti are provided by phosphorusfree membrane lipids, i.e., SL, OL, and DGTS (Geiger et al. 1999), and therefore, we wanted to study the potential role of phosphorus-free membrane lipids in the $S$. meliloti-alfalfa symbiosis. Mutants lacking SL, OL, or DGTS, the three possible double mutants deficient in two of the respective phosphorus-free membrane lipids, as well as the triple mutant SOD1, unable to synthesize OL, SL, or DGTS, were equally effective and competitive for the formation of nitrogen-fixing root nodules as the wild type. Therefore, the lack of any one or even all three of these phosphorus-free membrane lipids does not affect the symbiotic behavior of $S$. meliloti at all, not even the competitiveness of such a triple mutant strain, suggesting that there is no prominent role for the phosphorus-free membrane lipids SL, OL, or DGTS when $S$. meliloti lives inside its legume host and suggesting, further, that $S$. meliloti bacteria or bacteroids might not be limited for phosphorus when inside their host. In phoCDET mutants, which are deficient in a high-affinity phosphate transport system, the low-affinity phosphate transport system OrfA-Pit is repressed, and therefore, even during growth on phosphate-rich media, phoCDET mutants are starved for phosphate. In suppressor mutants of the phoCDET mutants, when OrfA-Pit is functional, phosphate assimilation and symbiosis is restored (Bardin et al. 1998). PhoB is a crucial regulator mediating phosphate stress responses in many bacteria. Mutants of S. meliloti (Al-Niemi et al. 1997b; Bardin and Finan 1998) or Bradyrhizobium japonicum (Minder et al. 1998) deficient in $p h o B$ form nitrogen-fixing nodules on their respective host plants, suggesting that adaptation to phosphate-limiting conditions is not needed in the bacteroid. Notably, some of the genes controlling SL biosynthesis $(s q d B)$ (Weissenmayer et al. 2000), OL biosynthesis (olsB = smc01127) (Gao et al. 2004), or DGTS biosynthesis (btaA and btaB $=s m c 1848$ and smcl847) are strongly induced in a phoB-dependent manner under phosphorus-limiting conditions (Krol and Becker 2004). Strikingly, there is very little overlap in global changes in protein expression profiles (Djordjevic et al. 2003) or of gene expression under symbiotic (Becker et al. 2004) or phosphate-starved conditions (Krol and Becker 2004), suggesting again that bacteroids of $S$. meliloti are not starved of phosphorus sources. Soybean nodules contain relatively large amounts of total phosphate
(Gaude et al. 2004), suggesting that there must be efficient mechanisms for transporting phosphorus sources from the plant to the bacterial microsymbiont. In the functional symbiosis between rhizobia and legumes, organic carbon sources are provided by the plant to the bacteroid, and in return, reduced nitrogen sources are provided by the bacteroids to the plant. Based on the results mentioned above, one might hypothesize that bacteroids are not limited by phosphorus because the plant provides sufficient usable phosphorus sources for the microsymbiont.

In free-living conditions, e.g., in defined media with growthlimiting amounts of phosphate, DGTS is the major lipid formed in wild-type $S$. meliloti, and btaA-deficient mutants unable to form DGTS compensate the lack of this lipid by forming more OL, SL, and PC. Notably, in DGTS-deficient mutants, OL is the major lipid formed under phosphate-limiting conditions. In btaA-olsA-deficient mutants that cannot form either DGTS or OL, a growth phenotype is observable, as they reach only lower growth yields under phosphate-limiting conditions of growth. In soils, concentration of inorganic phosphate is low $(0.1$ to $10 \mu \mathrm{M})$ (Bieleski 1973). We suggest that the ability to form phosphorus-free lipids such as DGTS or OL permits $S$. meliloti to grow to higher cell yields and therefore provides a significant advantage in phosphorus-poor environments.

\section{MATERIALS AND METHODS}

Bacterial strains, plasmids, and growth conditions.

The bacterial strains and plasmids used and their relevant characteristic are shown in Table 2. S. meliloti strains were grown either in complex tryptone yeast (TY) medium that contained $4.5 \mathrm{mM} \mathrm{CaCl}_{2}$ (Beringer 1974) or minimal medium (Sherwood 1970$)$ with succinate $(8.3 \mathrm{mM})$ replacing mannitol as the carbon source, at $29^{\circ} \mathrm{C}$ on a gyratory shaker. Growth rates in minimal medium containing either high $(1.3 \mathrm{mM})$ or low $(0.02 \mathrm{mM})$ concentrations of inorganic phosphate $(\mathrm{Pi})$ were determined as previously described (Weissenmayer et al. 2002).

E. coli strains were cultured on Luria-Bertani (LB) medium (Miller 1972) at $37^{\circ} \mathrm{C}$. Antibiotics were added to the media . when required. In the case of $S$. meliloti, the following 400; gentamicin, 70; piperacillin, 40; tetracycline, 4; and neomycin, 200. With E. coli, the following concentrations were added: spectinomycin, 200; carbenicillin, 100; tetracycline, 20; gentamicin, 10; and kanamycin, 50.

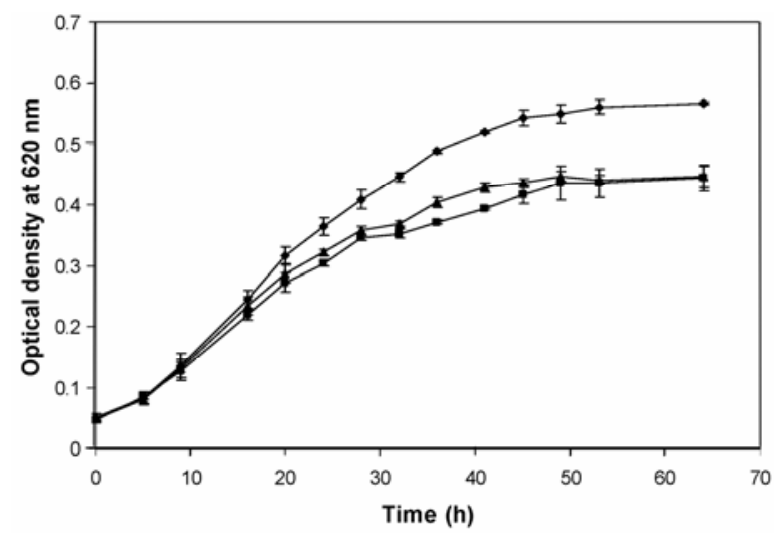

Fig. 6. Growth of Sinorhizobium meliloti 1021 wild type $(\bullet)$, mutant OD1 (A), and mutant SOD1 (ם) on Sherwood minimal medium containing growth-limiting concentrations $(0.02 \mathrm{mM})$ of inorganic phosphate. The values shown are mean values derived from three independent experiments and error bars represent \pm standard deviations. 
Plasmids pRK404, pMP3510, pJG03, pBW76, or suicide plasmid derivatives were mobilized into $S$. meliloti strains by triparental mating, using the mobilizing plasmid pRK2013 as described previously (Ruvkun and Ausubel 1981). The pGUS3 plasmid containing a $n f e D$-gusA fusion was used in competition assays (García-Rodríguez and Toro 2000).

\section{Cloning of the sinorhizobial $b t a A$ and $b t a B$ genes and inactivation of $b t a A$}

by a gentamicin resistance-conferring cassette.

Using polymerase chain reaction (PCR) and specific oligonucleotides (forward oEMM509: AAACTTCGACAAGC TTTTGAATGG and reverse oEMM508: AAA $\underline{\text { GGATCCAAT }}$ AGAAATGATAGGGCGCGT), a fragment of 3,210 bp that contains the complete open reading frames homologous to btaA and btaB from Rhodobacter sphaeroides (smc01848 and smc01847; coordinates $2,365,219$ to $2,368,428$ in the $S$. meliloti 1021 chromosome) was amplified from $S$. meliloti 1021 genomic DNA with Taq polymerase. Suitable restriction sites (underlined) for cloning of the fragment were either present in the genomic DNA (HindIII in forward primer) or introduced by PCR with the oligonucleotide (BamHI in reverse primer). After restriction with HindIII and BamHI, the PCRamplified DNA fragment was cloned into pUC19 vector to obtain plasmid pBW89. The HindIII-BamHI fragment of pBW89 was excised and recloned into the broad-host-range plasmid pRK404 to obtain plasmid pJG03 for use in S. meliloti.

For inactivation of the sinorhizobial btaA gene (SMCD1848), first the HindIII and BamHI fragment of pBW89 was cloned in vector pBBR1MCS (which does not contain a $B g l I I$ restriction site), to give plasmid pJG26. Next, the internal 316-bp $B g l$ II fragment of btaA was replaced by a $B a m \mathrm{HI}$ fragment containing the gentamicin omega interposon obtained from plasmid pAC $\Omega$ Gm (Schweizer 1993), resulting in plasmid pJG28. The XhoI fragment of pJG28 carrying the gentamicin-inactivated $b t a A$ gene was cloned into the SalI site of suicide vector pK18mobsacB (Schäfer et al. 1994) to yield pJG30. Via triparental mating using pRK2013 as a helper plasmid, pJG30 was introduced into the wild-type strain S. meliloti 1021. Transconjugants were selected on TY medium containing neomycin, gentamicin, and piperacillin to select for single recombinants in a first step. Single recombinants were grown under nonselective conditions in complex medium for 1 day before being plated on TY medium containing $10 \%$ (wt/vol) sucrose and gentamicin. Isolated colonies grew for 5 days, and membrane lipids of 14 neomycin-sensitive candidates were analyzed (data not shown). In contrast to the wild-type strain, none of the selected candidates formed DGTS under phosphate limitation. Southern blot analysis confirmed that the DGTSdeficient strains were indeed double recombinants in which an internal fragment of the $b t a A$ gene was replaced by a cassette conferring gentamicin resistance (data not shown). Using restriction analysis of plasmid pJG28, the orientation of the gentamicin resistance cassette was determined to be in opposite orientation to the btaA-interrupted gene in mutant DGTS1.

\section{Cloning of the sinorhizobial $b t a A$ and $b t a B$ genes \\ for expression in $E$. coli.}

Using PCR and specific oligonucleotides (forward oEMM801 GGAATACATATGACCGACTTCGCCCCGGAT and reverse oEMM804 AAAGGATCCCTACGGTGCCGCGCGGCGATA GA), the coding region for SMc01848 and SMc01847 was amplified from $S$. meliloti 1021 genomic DNA with the GeneAmp PCR amplification kit (Applied Biosystems, Branchburg, NJ, U.S.A.). Suitable restriction sites (underlined) for cloning were introduced by PCR with the oligonucleotides ( NdeI in forward primer and BamHI in reverse primer). After restriction with
NdeI and BamHI, the PCR-amplified DNA fragment was cloned into a pET9a vector (Studier et al. 1990), to obtain the expression plasmid pIML05 for use in E. coli.

\section{Construction of $S$. meliloti mutants deficient in two or three phosphorus-free membrane lipids.}

General transduction of the respective cassette-inactivated genes using the phage ФM12 was performed similarly to that described previously (Finan et al. 1984). By ФM12 transduction, the sqdB::kan mutation from $S$. meliloti SLD11 (Weissenmayer et al. 2000) was transferred to wild-type $S$. meliloti 1021 to obtain the SL-deficient mutant SLD12. Inactivation of olsA with a spectinomycin resistance cassette was done in a way similar to that described for the inactivation of ols $A$ with a kanamycin resistance cassette (Weissenmayer et al. 2002). In short, pBW63 was cut with AgeI and filled in, and the spectinomycin resistance-conferring 2.0-kb SmaI-SmaI fragment of pHY109 (Østerås et al. 1998) was inserted in the opposite orientation to olsA, giving rise to $\mathrm{pBW} 71$. The $5.2-\mathrm{kb}$ $X b a \mathrm{I}-K p n I$ fragment of pBW71 was cloned into the broadhost-range vector pMP3510, resulting in plasmid pBW76. The plasmid pBW76 was mobilized into wild-type $S$. meliloti 1021. The spectinomycin-inactivated olsA was recombined into the wild-type genome by the plasmid-incompatibility technique, as described previously (Østerås et al. 1998). Potential double recombinants were tetracycline-sensitive and were analyzed further by Southern hybridization (data not shown). The olsA-deficient strain was designated ORLD8 and was unable to form OL. Double knock-out mutants and the triple mutant were constructed by general transduction of the respective cassette-inactivated genes, using the phage $\Phi \mathrm{M} 12$ similarly to the way described previously (Finan et al. 1984).

\section{In vivo labeling of bacterial strains} with $\left[{ }^{14} \mathrm{C}\right]$ acetate and quantitative analysis of lipid extracts.

The lipid compositions of S. meliloti 1021 wild-type and mutant strains and complemented mutants were determined following labeling with $\left[1-{ }^{14} \mathrm{C}\right]$ acetate. Cultures $(1 \mathrm{ml})$ in minimal medium were inoculated from precultures grown in the same medium. After the addition of $2 \mu \mathrm{Ci}\left[1-{ }^{14} \mathrm{C}\right]$ acetate $(60$ $\mathrm{mCi} / \mathrm{mmol}$ ) to each culture, the cultures were incubated for 24 $\mathrm{h}$ if grown on high phosphate or $30 \mathrm{~h}$ if grown on low phosphate.

For detection of product formation after overexpression of $\mathrm{SMc} 01848$ and SMc01847 in E. coli, derivaties of strain BL21(DE3) pLysS were cultivated in complex LB medium (1 $\mathrm{ml}$ ) and at densities of $4 \times 10^{8}$ cells per milliliter, $0.1 \mathrm{mM}$ isopropyl- $\beta$-D-thiogalactoside and $2 \mu \mathrm{Ci} \quad\left[1-{ }^{14} \mathrm{C}\right]$ acetate $(60$ $\mathrm{mCi} / \mathrm{mmol}$ ) were added, and cultures were incubated for another $3 \mathrm{~h}$ at 30 or $37^{\circ} \mathrm{C}$.

Cells were harvested by centrifugation, were washed with $500 \mu \mathrm{l}$ of water, and were resuspended in $100 \mu \mathrm{l}$ of water. The lipids were extracted according to Bligh and Dyer (1959). The chloroform phase was used for lipid analysis on TLC plates and after one- (Weissenmayer et al. 2002) or two-dimensional (Geiger et al. 1999) separation, the individual lipids were quantified as described by de Rudder and associates (1997).

\section{Mass spectrometric analysis.}

For spectrometric analysis of lipids after overexpression of SMc01848 and SMc01847 in E. coli, derivatives of strain BL21(DE3) pLysS were cultivated in complex LB medium (1 liter) and at densities of $4 \times 10^{8}$ cells per milliliter, $0.1 \mathrm{mM}$ isopropyl- $\beta$-D-thiogalactoside was added, and cultures were incubated for another $4 \mathrm{~h}$ at $37^{\circ} \mathrm{C}$. Cells were harvested by centrifugation and the lipids were extracted according to Bligh and Dyer (1959). The chloroform phase was analyzed. 
ES-MS and CID MS/MS were obtained using a Micromass Q-ToF (time of flight) 2 hybrid quadrupole orthogonal ToF mass spectrometer (Altrincham, U.K.) operated in the positive ion mode. The instrument was fitted with a Micromass Z-spray electrospray source. A fused silica capillary was used to deliver the sample to the ion source in a continuous flow at $1 \mu \mathrm{l}$ $\min ^{-1}$ controlled by a syringe driver. The instrument was operated with a capillary voltage of $3,500 \mathrm{~V}$, the sampling cone was held at $47 \mathrm{~V}$, with a source temperature of $80^{\circ} \mathrm{C}$. ToF mass spectra were integrated every $2.4 \mathrm{~s}$ over a range of $\mathrm{m} / \mathrm{z} .50$ to 1,500. In the MS/MS mode, the collision energy offset was varied between settings of 4 to $80 \mathrm{~V}$. Argon was used as the collision gas, detection of the ions was performed using a dual microchannel plate detector system, and the data system used was Windows NT-based Micromass MassLynx version 3.4.

\section{Plant assays.}

Alfalfa (Medicago sativa L.) plants were grown in a nitrogen-free medium as described by Olivares and associates (1980), which contained about $0.7 \mathrm{mM}$ phosphate. To test the infectivity of the rhizobial strains, 24 individual plants were inoculated with $10^{6}$ cells. After inoculation, the number of nodulated plants and the number of nodules per plant were recorded daily. To determine the competitive ability, 12 plants were inoculated with $10^{6}$ cells corresponding to either $1021 \times$ 1021 (pGUS3) or SOD1 $\times 1021$ (pGUS3) mixtures in the ratio of $1: 1$. The plasmid pGUS3 contains the marker gene coding for $\beta$-glucuronidase. To determine nodule occupancy, roots were collected 12 days after inoculation, were washed briefly with water, and were incubated overnight in the dark at $37^{\circ} \mathrm{C}$ in $1 \mathrm{mM} \mathrm{X}$-Gluc (5-bromo-chloro-3-indolyl- $\beta$-D-glucuronide, Apollo Scientific, Bredbury, U.K.) in $50 \mathrm{mM}$ sodium phosphate buffer ( $\mathrm{pH} 7.5$ ) with $1 \%$ sodium dodecyl sulfate. Those nodules occupied by 1021 (pGUS3) stain blue, whereby nodule occupancy could be determined by counting blue and white nodules.

\section{ACKNOWLEDGMENTS}

This article is dedicated to Franz Lingens on the occasion of his 80th birthday. This work was supported by grants from DGAPA/UNAM (IN200802), the Consejo Nacional de Ciencia y Tecnología de México (CONACyT 36274-N and 42578/A-1), as well as the Howard Hughes Medical Institute (HHMI 55003675). J. Thomas-Oates gratefully acknowledges financial support from the Analytical Chemistry Trust Fund, the RSC Analytical Division, and the EPSRC, and financial support (to S. J. Gaskell and R. J. Beynon) from the Higher Education Funding Council for England, with additional support from Smith Kline Beecham, UMIST, University of Wales College of Medicine, and Chugai Pharmaceuticals, for the purchase of the Q-ToF (located at UMIST).

\section{LITERATURE CITED}

Al-Niemi, T. S., Kahn, M. L., and McDermott, T. R. 1997A. Characterization of phosphorus metabolism in the bean-Rhizobium tropici symbiosis. Plant Physiol. 113:1233-1242.

Al-Niemi, T. S., Summers, M. L., Ellins, J. G., Kahn, M. L., and McDermott, T. R. 1997B. Regulation of the phosphate stress response in $R$. meliloti by PhoB. Appl. Environ. Microbiol. 63:4978-4981.

Bardin, S., Dan, S., Osteras, M., and Finan, T. M. 1996. A phosphate transport system is required for symbiotic nitrogen fixation by Rhizobium meliloti. J. Bacteriol. 178:4540-4547.

Bardin, S. D. and Finan, T. M. 1998. Regulation of phosphate assimilation in Rhizobium (Sinorhizobium) meliloti. Genetics 148:1689-1700.

Bardin, S. D., Voegele, R. T., and Finan, T. M. 1998. Phosphate assimilation in Rhizobium (Sinorhizobium) meliloti: Identification of a pit-like gene. J. Bacteriol. 180:4219-4226.

Becker, A., Berges, H., Krol, E., Bruand, C., Rüberg, S., Capela, D., Lauber, E., Meilhoc, E., Ampe, F., de Bruijn, F. J., Fourment, J., Francez-Charlot, A., Kahn, D., Küster, H., Liebe, C., Pühler, A., Weidner, S., and Batut, J. 2004. Global changes in gene expression in
Sinorhizobium meliloti 1021 under microoxic and symbiotic conditions. Mol. Plant-Microbe Interact. 17:292-303.

Benning, C., Huang, Z.-H., and Gage, D. A. 1995. Accumulation of a novel glycolipid and a betaine lipid in cells of Rhodobacter sphaeroides grown under phosphate limitation. Arch. Biochem. Biophys. 317:103111.

Beringer, J. E. 1974. R factor transfer in Rhizobium leguminosarum. J. Gen. Microbiol. 84:188-198.

Bieleski, R. L. 1973. Phosphate pools, phosphate transport, and phosphate availability. Annu. Rev. Plant Physiol. 24:225-252.

Bligh, E. G., and Dyer, J. W. 1959. A rapid method of total lipid extraction and purification. Can. J. Biochem. Physiol. 37:911-917.

Ditta, G., Schmidhauser, T., Yacobson, E., Lu, P., Liang, X.-W., Finlay, D. R., Guiney, D., and Helinski, D. R. 1985. Plasmids related to the broad host range vector, pRK290, useful for gene cloning and for monitoring expression. Plasmid 13:149-153.

Djordjevic, M. A., Chen, H. C., Natera, S., Van Noorden, G., Menzel, C., Taylor, S., Renard, C., Geiger, O., The Sinorhizobium DNA Sequencing Consortium, and Weiller, G. F. 2003. A global analysis of protein expression profiles in Sinorhizobium meliloti: Discovery of new genes for nodule occupancy and stress adaptation. Mol. Plant-Microbe Interact. 16:508-524.

Dörmann, P., and Benning, C. 2002. Galactolipids rule in seed plants. Trends Plant Sci. 7:112-118.

Figurski, D. H., and Helinski, D. R. 1979. Replication of an origin-containing derivative of plasmid RK2 dependent on a plasmid function provided in trans. Proc. Natl. Acad. Sci. U.S.A. 76:1648-1652.

Finan, T. M., Hartwieg, E., LeMieux, K., Bergman, K., Walker, G. C., and Signer, E. R. 1984. General transduction in Rhizobium meliloti. J. Bacteriol. 159:120-124.

Galibert, F., Finan, T. M., Long, S. R., Puhler, A., Abola, P., Ampe, F., Barloy-Hubler, F., Barnett, M. J., Becker, A., Boistard, P., Bothe, G., Boutry, M., Bowser, L., Buhrmester, J., Cadieu, E., Capela, D., Chain, P., Cowie, A., Davis, R. W., Dreano, S., Federspiel, N. A., Fisher, R. F., Gloux, S., Godrie, T., Goffeau, A., Golding, B., Gouzy, J., Gurjal, M., Hernandez-Lucas, I., Hong, A., Huizar, L., Hyman, R. W., Jones, T., Kahn, D., Kahn, M. L., Kalman, S., Keating, D. H., Kiss, E., Komp, C., Lelaure, V., Masuy, D., Palm, C., Peck, M. C., Pohl, T. M., Portetelle, D., Purnelle, B., Ramsperger, U., Surzycki, R., Thebault, P., Vandenbol, M., Vorholter, F. J., Weidner, S., Wells, D. H., Wong, K., Yeh, K. C., and Batut, J. 2001. The composite genome of the legume symbiont Sinorhizobium meliloti. Science:293:668-72.

Gao, J.-L., Weissenmayer, B., Taylor, A. M., Thomas-Oates, J., LópezLara, I. M., and Geiger, O. 2004. Identification of a gene required for the formation of lyso-ornithine lipid, an intermediate in the biosynthesis of ornithine-containing lipids. Mol. Microbiol. 53:1757-1770.

Gaude, N., Tippmann, H., Flemetakis, E., Katinakis, P., Udvardi, M., and Dörmann, P. 2004. The galactolipid digalactosyldiacylglycerol accumulates in the peribacteroid membrane of nitrogen-fixing nodules of soybean and Lotus. J. Biol. Chem. 279:34624-34630.

García-Rodríguez, F. M., and Toro, N. 2000. Sinorhizobium meliloti nfe (nodulation formation efficiency) genes exhibit temporal and spatial expression patterns similar to those of genes involved in symbiotic nitrogen fixation. Mol. Plant-Microbe Interact. 13:583-591.

Geiger, O., Röhrs, V., Weissenmayer, B., Finan, T. M., and Thomas-Oates, J. E. 1999. The regulator gene $p h o B$ mediates phosphate stress-controlled synthesis of the membrane lipid diacylglyceryl- $N, N, N$-trimethylhomoserine in Rhizobium (Sinorhizobium) meliloti. Mol. Microbiol. 32:63-73.

Hanahan, D. 1983. Studies on transformation of Escherichia coli with plasmids J. Mol. Biol. 166:557-580.

Klug, R. M., and Benning, C. 2001. Two enzymes of diacylglyceryl-O-4'$(N, N, N$,-trimethyl)homoserine biosynthesis are encoded by btaA and btaB in the purple bacterium Rhodobacter sphaeroides. Proc. Natl. Acad. Sci. U.S.A. 98:5910-5915.

Kovach, M. E., Phillips, R. W., Elzer, P. H., Roop, R. M., II, and Peterson, K. M. 1994. pBBR1MCS: A broad-host-range cloning vector. BioTechniques 16:800-802.

Krol, E., and Becker, A. 2004. Global transcriptional analysis of the phosphate starvation response in Sinorhizobium meliloti strains 1021 and 2011. Mol. Gen. Genomics 272:1-17.

López-Lara, I. M., Sohlenkamp, C., and Geiger, O. 2003. Membrane lipids in plant-associated bacteria: Their biosyntheses and possible functions. Mol. Plant-Microbe Interact. 16:567-579.

Meade, H. M., Long, S. R., Ruvkun, G. B., Brown, S. E., and Ausubel, F. M. 1982. Physical and genetic characterization of symbiotic and auxotrophic mutants of Rhizobium meliloti induced by transposon Tn5 mutagenesis. J. Bacteriol. 149:114-122.

Miller, J. H. 1972. Experiments in Molecular Genetics. Cold Spring Harbor Laboratory, Cold Spring Harbor, NY, U.S.A. 
Minder, A. C., Narberhaus, F., Fischer, H.-M., and Hennecke, H. 1998. The Bradyrhizobium japonicum $p h o B$ gene is required for phosphatelimited growth but not for symbiotic nitrogen fixation. FEMS (Fed. Eur. Microbiol. Soc.) Lett. 161:47-52.

Minnikin, D. E., and Abdolrahimzadeh, H. 1974. The replacement of phosphatidylethanolamine and acidic phospholipids by an ornithineamide lipid and a minor phosphorous-free lipid in Pseudomonas fluorescens NCMB 129. FEBS (Fed. Eur. Biochem. Soc.) Lett. 43:257-260.

Minnikin, D. E., Abdolrahimzadeh, H., and Baddiley, J. 1972. Variation of polar lipid composition of Bacillus subtilis Marburg with different growth conditions. FEBS (Fed. Eur. Biochem. Soc.) Lett. 27:16-18.

Minnikin, D. E., Abdolrahimzadeh, H., and Baddiley, J. 1974. Replacement of acidic phospholipids by acidic glycolipids in Pseudomonas diminuta. Nature (London) 249:268-269.

Olivares, J., Casadesús, J., and Bedmar, E. J. 1980. Method for testing degree of infectivity of Rhizobium meliloti strains. Appl. Environ. Microbiol. 39:967-970.

Østerås, M., Boncompagni, E., Vincent, N., Poggi, M. C., and Le Rudulier, D. 1998. Presence of a gene encoding choline sulfatase in Sinorhizobium meliloti bet operon: Choline- $O$-sulfate is metabolized into glycine betaine. Proc. Natl. Acad. Sci. USA 95:11394-11399.

de Rudder, K. E. E., Thomas-Oates, J. E., and Geiger, O. 1997. Rhizobium meliloti mutants deficient in phospholipid $N$-methyltransferase still contain phosphatidylcholine. J. Bacteriol. 179:6921-6928.

Ruvkun, G. B., and F. M. Ausubel, F. M. 1981. A general method for sitedirected mutagenesis in procaryotes. Nature (London) 289:85-88.

Schäfer, A., Tauch, A., Jäger, W., Kalinowski, J., Thierbach, G., and Pühler, A. 1994. Small mobilizable multi-purpose cloning vectors derived from the Escherichia coli plasmids pK18 and pK19: Selection of defined deletions in the chromosome of Corynebacterium glutamicum. Gene 145:69-73.

Schweizer, H. D. 1993. Small broad-host-range gentamicin resistance gene cassettes for site-specific insertion and deletion mutagenesis. BioTechniques 15:831-834.

Sherwood, M. T. 1970. Improved synthetic medium for the growth of $R h i$ zobium. J. Appl. Bacteriol. 33:708-713.

Spaink, H. P., Wijfjes, A. H. M., and Lugtenberg, B. J. J. 1995. Rhizobium NodI and NodJ proteins play a role in the efficiency of secretion of lipochitin oligosaccharides. J. Bacteriol. 177:6276-6281.

Studier, F. W., Rosenberg, A. H., Dunn, J. J., and Dubendorff, J. W. 1990. Use of T7 RNA polymerase to direct expression of cloned genes. Methods Enzymol. 185:60-89.

Weissenmayer, B., Geiger, O., and Benning, C. 2000. Disruption of a gene essential for sulfoquinovosyldiacylglycerol biosynthesis in Sinorhizo bium meliloti has no detectable effect on root nodule symbiosis. Mol. Plant-Microbe Interact. 13:666-672.

Weissenmayer, B., Gao, J.-L., López-Lara I. M., and Geiger O. 2002. Identification of a gene required for the biosynthesis of ornithinederived lipids. Mol. Microbiol. 45:721-733.

Yanisch-Perron, C., Vieira, J., Messing, J. 1985. Improved M13 phage cloning vectors and host strains: Nucleotide sequences of the M13mp18 and pUC19 vectors. Gene 33:103-119.

\section{AUTHOR-RECOMMENDED INTERNET RESOURCE}

Sinorhizobium meliloti strain 1021 Genome Project website: histone toulouse.inra.fr/bioinfo/annotation/iANT/bacteria/rhime/index.html) 